\section{Larynx-Ca: Organerhaltende Therapie schmälert Überlebenschancen nicht}

\author{
Moderne Therapieverfahren ermöglichen den Erhalt des Kehlkopfs auch bei \\ lokal fortgeschrittenen Larynxkarzinomen. Bedenken, die Sterblichkeit könne \\ dabei im Vergleich zur Laryngektomie steigen, haben sich in einer US-Studie \\ nicht bestätigt.
}

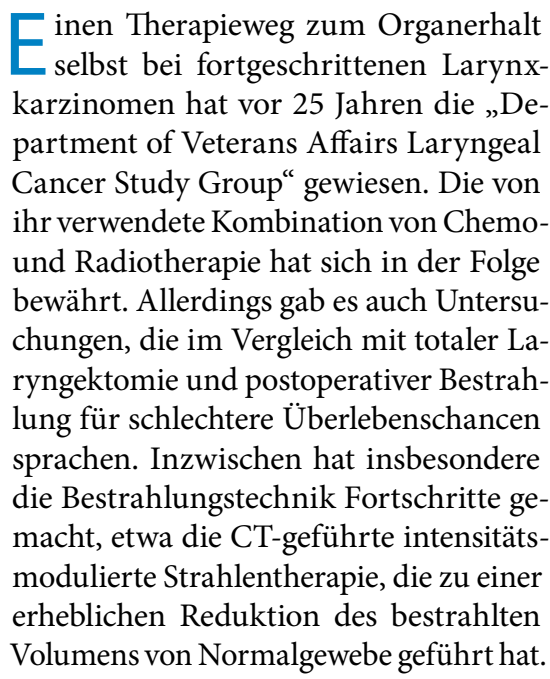

Ein Forscherteam um Clifton Fuller vom University of Texas MD Anderson Cancer Center in Houston hat die Behandlungsergebnisse von 412 Patienten mit T3N0-3M0-Plattenepithelkarzinomen des Larynx aus den Jahren 1985 bis 2011 verglichen, die sich verschiedenen Therapieverfahren unterzogen hatten: 166 erhielten eine larynxerhaltende Chemoradiotherapie (LE-CRT), 122 eine larynxerhaltende Radiotherapie (LE-RT), und 125 unterzogen sich einer totalen Laryngektomie mit postoperativer Bestrahlung (TL-PORT).

Die Fünf- bzw. Zehn-Jahres-Überlebensraten fielen in der LE-CRT-Gruppe am höchsten aus (67\% bzw. $43 \%$ ), gefolgt von der TL-PORT-Gruppe $(50 \%$ bzw. $35 \%$ ) und der LE-RT-Gruppe (46\% bzw. $25 \%)$, ein signifikantes Ergebnis ( $\mathrm{p}=$ $0,006)$. Gemessen am Anteil der lokoregionären Tumorkontrolle über zehn Jahre hinweg war TL-PORT statistisch signifikant besser als das LE-RT-Verfahren ( $83 \%$ vs. $64 \%, p=0,003)$, die Differenz zur LE-CRT-Gruppe hingegen war statistisch unbedeutend ( $83 \%$ vs. $77 \%, \mathrm{p}=0,2)$. Mindestens zehn Jahre frei von Metastasen blieben $87 \%$ der Patienten aus der LECRT-Gruppe, $90 \%$ aus LE-RT und $71 \%$ aus TL-PORT.

Fazit: Die Aussage von Fuller und Kollegen fällt eindeutig aus: „Für Patienten mit T3-Larynxkrebs führt LE-CRT zu besseren funktionellen, onkologischen und Überlebensergebnissen als TLPORT und LE-RT.“

Dr. Robert Bublak

Fuller $\mathrm{CD}$ et al. Long-term outcomes after multidisciplinary management of $\mathrm{T} 3$ laryngeal squamous cell carcinomas: Improved functional outcomes and survival with modern therapeutic approaches. Head Neck 2016, online 28. Juli

der den RSDI-Score noch die genannten sekundären Erfolgsparameter.

Fazit: Laut den Studienautoren um Paul Little von der Universität in Southampton resultierte die Empfehlung, täglich Nasenspülungen mit Kochsalzlösung vorzunehmen, in einer „bescheidenen“ Verbesserung der gesundheitsbezogenen Lebensqualität bei Rhinosinusitis. Der Effekt war geringer als aufgrund früherer Studien zu erwarten war. Diese Untersuchungen mit weniger Teilnehmern hätten allerdings eine intensive Betreuung der Patienten beinhaltet, während in der aktuellen Studie die Anleitung zur Nasenspülung nur über ein kurzes Video erfolgte. Außerdem seien Nasenduschen in den USA, wo zwei der Studien durchgeführt wurden, extrem populär. Beides habe vermutlich die positive Erwartungshaltung gegenüber der Intervention verstärkt.

Dr. Beate Schumacher

Little P et al. Effectiveness of steam inhalation and nasal irrigation for chronic or recurrent sinus symptoms in primary care: a pragmatic randomized controlled trial. CMAJ 2016, online 18. Juli 